\title{
Temporal Transitions of Demographic Dot Maps
}

\author{
Jeff Allen \\ Department of Geography \& Planning \\ University of Toronto \\ jeff.allen@utoronto.ca
}

\begin{abstract}
Dot maps are often used to display the distributions of populations over space. This paper details a method for extending dot maps in order to visualize changes in spatial patterns over time. Specifically, we outline a selective linear interpolation procedure to encode the time range in which dots are visible on a map, which then allows for temporal queries and animation. This methodology is exemplified first by animating population growth across the United States, and second, through an interactive application showing changing poverty distributions in Toronto, Canada.
\end{abstract}

\section{Keywords}

Dot maps; demography; time; interpolation

This manuscript preprint version is made available under the CC-BY-NC-ND 4.0 license:

http://creativecommons.org/licenses/by-nc-nd/4.0/

It has since been published in the International Journal of Cartography:

https://doi.org/10.1080/23729333.2021.1910184 


\section{Introduction}

Analyzing demographic patterns across space and over time are important tasks for a wide range of researchers in fields including, but not limited to, human geography, urban planning, epidemiology, political science, history, and sociology (Ades, Apparicio, \& Seguin, 2012; Delmelle, 2017; Franklin, 2019; Logan, Xu, \& Stults, 2014; St-Hilaire, Moldofsky, Richard, \& Beaudry, 2007). Temporal demographic analysis, however, is often constrained to specific data collection years and data is often only available as aggregated to pre-defined spatial units. This limits visualizations of changing demographic patterns to these spatial and temporal constraints.

A solution to these constraints are temporal dot maps. Dot maps are often used to visualize distributions and clustering of different demographic groups over space. Common applications of dot maps include visualizing the spatial distributions of categorical population data such as educational attainment (Walker, 2018), poverty (Barreto, 1993), or race and ethnicity (Cable, 2013). Dot maps are often preferred over areal choropleth maps because of their ability to display and convey both densities and proportions (Dent, Torguson, \& Hodler, 1990; Dorling, 1993; Walker, 2018).

Accordingly, the objective of this paper is to develop and exemplify a method for interpolating demographic dot density maps so they can display changes over time. This includes creating static dot maps, followed by a description of a novel selective linear interpolation procedure which encodes the time range in which dots are visible on a map. This encoding allows for querying and animating changes over time without generating dots for periods in which there are not known data. It also allows for smoother transitions. This procedure is exemplified for two use case scenarios, the first animating population growth in the United States from 1900 to 2000, and the second an interactive application showing changing poverty distributions in Toronto, Canada from 1991 to 2016.

\section{Background}

\subsection{Spatiotemporal Demographic Analysis}

Researchers have quantitatively studied human populations for thousands of years. The term "demography" stems from ancient Greece, where some of the earliest known population censuses were recorded (Missiakoulis, 2010). (demo $=$ the people, graphy $=$ description or measurement). In the modern era, analyzing demographic data, in particular, examining changing spatial patterns of populations over time, is conducted by researchers across public, academic, and private sectors for a variety of research purposes. Common analyses include, for example, describing spatial patterns of national population growth (St-Hilaire et al., 2007), forecasting future growth patterns (Chi \& Voss, 2011), examining trends of population decline (Franklin, 2019), quantifying urban sprawl (Sun, 2019), analyzing neighbourhood demographic change (Delmelle, 2017), or uncovering trends of suburbanization of poverty (Ades et al., 2012).

Demographic analysis is predominantly based on data from national censuses or other administrative surveys. Contemporary national censuses are typically collected at regular intervals. In some nations, this is every 5 years (e.g. Canada, Ireland), in other nations, every 10 years (e.g. United States, United Kingdom). As such, analysis of census data is limited to these data collection periods, and any intermediate year analyses have to rely on interpolation processes (Fukuda, 2010).

Another major constraint of census data, as well as many other demographic data sources, are their representative geographical units. Because of privacy concerns, data are usually only released publicly as pre-aggregated to pre-defined spatial units. These can range from nation-wide administrative districts 
(e.g. states, provinces, counties, etc.) down to block level boundaries (e.g. the area enclosed by a typical city block). However, the smallest units often do not include the full range of data collected by the census, again because of privacy concerns. For example in Canada, block-sized polygons only include population and dwelling counts, while other variables have to be analyzed at larger, neighbourhood sized, spatial units (Statistics Canada, 2016). These spatial constraints result in difficulty to discern how patterns vary at regions or scales in which data are unavailable. As well, analysis bounded by specific geographic units can result in uncertainty caused by the modifiable areal unit problem, which is a source of statistical bias which occurs when individual level data are aggregated into polygons (Gehlke \& Biehl, 1934; Openshaw, 1979). Moreover, census boundaries can also change over time, meaning researchers often undertake labourious and error inducing areal interpolation processes to harmonize data to the same spatial units in order to conduct temporal analyses (Allen \& Taylor, 2018; Holt, Lo, \& Hodler, 2004; Logan et al., 2014; Schroeder, 2007).

Despite these spatial and temporal constraints, areal units such as neighbourhood boundaries remain the standard for visualization and geographic analysis of how places change over time. As such, by far the most common method for mapping demographic data is to use choropleths. Choropleth maps shade spatial units according to their associated attributes (Dent et al., 1990). They are successful at indicating patterns across an entire region (e.g. at a zoomed out view), or when wanting to locate values for specific administrative units (Lewandowsky et al., 1993). However, they are limited in that they are unable to show both proportions and densities, nor do they show any variations within each polygon, which can also give false impressions near the boundaries of spatial units (Dent et al., 1990).

\subsection{Dot Maps}

There are a number of alternative methods in which cartographers have mapped demographic data (Dent et al., 1990; Dorling, 1993). One of the most widely used alternatives are dot maps. Dot maps, also called dot density maps or dot distribution maps, use points to indicate the distribution of phenomena over geographic space. They are a kind of unit visualization, where observations are visually presented with individual points, rather than aggregated into groups such as polygons (Park, Drucker, Fernandez, \& Elmqvist, 2018). A comparison of a dot map and a choropleth map for the United States is displayed in Figure 1.

Dot maps can convey patterns of clustering and dispersion via the law of proximity, a Gestalt principle of organization, in which objects that are closer together are perceived as belonging to a group (Chen, 2010; MacEachren, 2004). On demographic dot maps, a collection of nearby dots indicate concentrations of populations such as cities, areas of high density within cities, or other congregations of high activity. Dot maps can also allow for viewing clusters of multiple sizes and at different scales. This is illustrated on the top of Figure 1 where we can observe an overall pattern of higher concentrations of population in the northeast of the USA compared to central parts of the country. Furthermore, within the northeast, we can observe how these populations are clustered and shaped to form specific cities and metropolitan regions, with particularly high concentrations along the eastern seaboard.

Dot maps are also often used to displayed categorical demographic data. Common uses include mapping race or ethnicity (Cable, 2013; Dmowska \& Stepinski, 2019), educational attainment (Walker, 2018), or socio-economic status (Barreto, 1993). These maps either present categorical variables in individual maps and then compare them as small multiples (Barreto, 1993), or on the same map via leveraging a qualitative colour scheme (Cable, 2013; Walker, 2018). The latter are able to convey relative proportions of groupings as well as patterns of clustering or dispersion. For example, a categorical dot a map of race in the United States is able to display patterns of racial segregation as well as overall concentrations of population groups in different areas (Cable, 2013). 


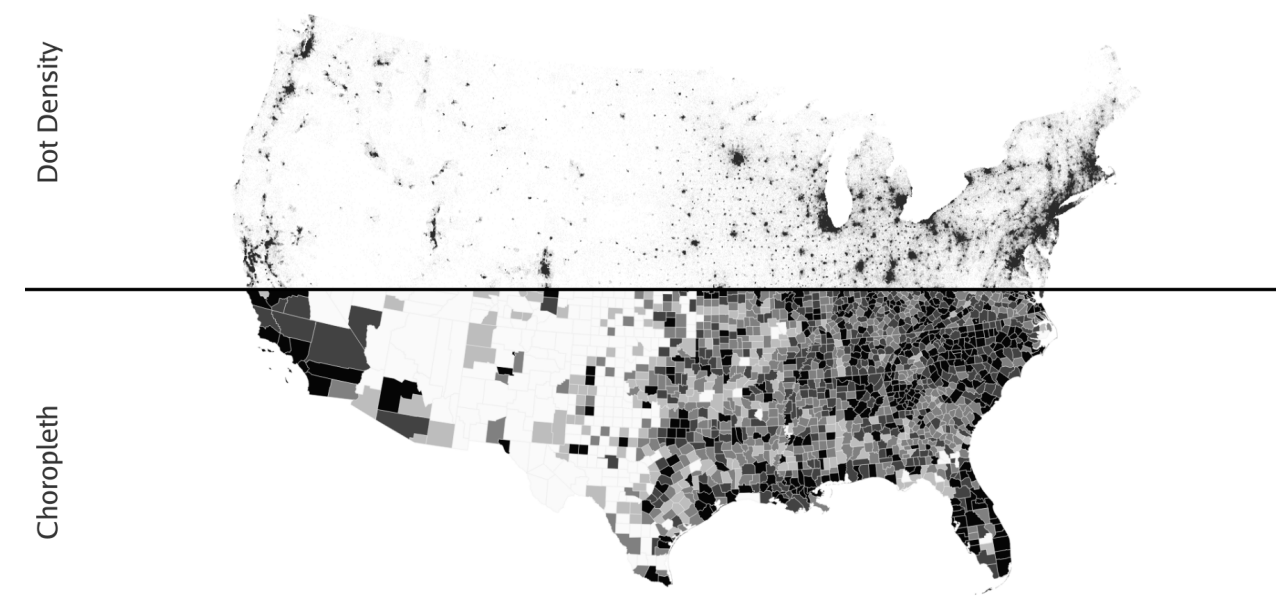

Figure 1: Comparison between a dot density map and county-level choropleth map showing population density in the United States (2000)

The documented history of dot maps dates back to the early 19th century. Examples of historical dot maps include national population distribution maps of France (Frere de Montizon, 1830; Konvitz, 1987) and the United Kingdom (Dorling, 1993; Petermann, 1852). Early research on dot maps was generally concerned about strategies for placing dots on dot maps. This included techniques for specifying the size of points, selecting the number of person per dot, and estimating coordinates of dots in their representative zones (Birch, 1938; Mackay, 1949). Much of this early literature focused on manual drafting techniques (Birch, 1938). More recently, computer applications have been developed to computationally generate dot maps given inputs of polygons and associated counts of populations (Hey, 2012; Hey \& Bill, 2014; Kimerling, 2009). With the advent of interactive web maps, recent research has also focused on how dot maps can be scaled, on-the-fly, to display an appropriate number of dots at different zoom levels (Walker, 2018; Zhang, Zhu, Xiao, \& Zhang, 2016). Another strand of recent research is to improve the estimation of dot location via dasymetric mapping, a technique which uses auxiliary data to weight where wthin a zone observations are more likely to occur (Dmowska \& Stepinski, 2019; Holt et al., 2004; Petrov, 2012). Auxiliary land use data can be of several forms, such as from a municipality's zoning or land use plans, derived from satellite imagery, or from population counts linked to smaller census boundaries.

Mapping temporal phenomena is also an ongoing topic for research and practice in cartography and visualization (MacEachren \& DiBiase, 1991; Vasiliev, 2006). However, there has been very limited use of dot maps to visualize change over time, despite discussion regarding the complications of visualizing spatial data that are constrained to specific data collection periods (Monmonier, 1996). There is one study in Los Angeles which used dot maps to represent change in demographics over time (Turner \& Allen, 2010). This study used dots on a static map to represent population loss and gain rather than people at an individual point in time (e.g. instead of 1 dot pertaining to 100 people, 1 dot pertained to 100 people leaving an area over a period of time) (Turner \& Allen, 2010). However, this method is limited to analyzing change for between only two time periods. There has been research on transitioning between other types of unit visualizations, such as scatter plots (Chevalier, Dragicevic, \& Franconeri, 2014; Park et al., 2018), but such transitions have not been applied to temporal dot maps. Overall, to date, there is a dearth of research on analyzing how dot density maps can be extended for mapping and visualizing spatio-temporal changes in demographic patterns. 


\section{Temporal Interpolation of Dot Maps}

The following outlines a procedure for generating dot maps for temporal visualizations. This process presumes that the input data are initially aggregated to spatial units, and that there are two or more time periods, $t^{*}$, in which there are known data for at specified spatial units. This is the predominant case when using demographic data sources such as national censuses.

The first step in this procedure is to estimate the coordinates of dots for the time periods in which we have known data for (e.g. for different census data collection periods). As mentioned in the previous section, there is a wide literature regarding selecting the locations of coordinates of dots on dot maps. These methods vary in sophistication from placing dots at purely random locations within a zone, placing dots in a way where a minimal spacing between dots is achieved, or using a dasymetric mapping technique to place dots in locations based on auxiliary data sources (Dmowska \& Stepinski, 2019; Hey \& Bill, 2014; Kimerling, 2009; Mackay, 1949). Figure 2 schematically shows different strategies for dot placement. This figure also shows how colour can be used to ascertain between two demographic categories. Part of the dot creation procedure also includes deciding how many people are represented by each dot. This decision is based on the scale of the map, number of categories, and how clustered observations are over space (Walker, 2018; Zhang et al., 2016). We also recommend that the method of dot placement be selected based on the targeted use of the map, made in conjunction with selecting the size of dots, as well as being mindful of available data and resources. For example, detailed land use data to perform dasymetric dot placement is more likely to be readily available for contemporary mapping projects than historical ones. This is because older land use or census boundary data required for dasymetric mapping are often hard to come by in digital formats (Allen \& Leahey, 2016). The two use case scenarios in the following section provide some further details on dot placement for specific data sources.

a) Random Dot Placement

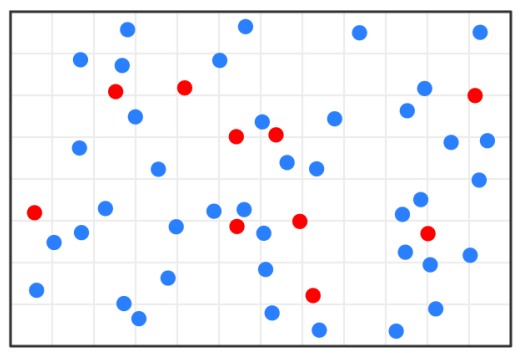

b) Land Use Data

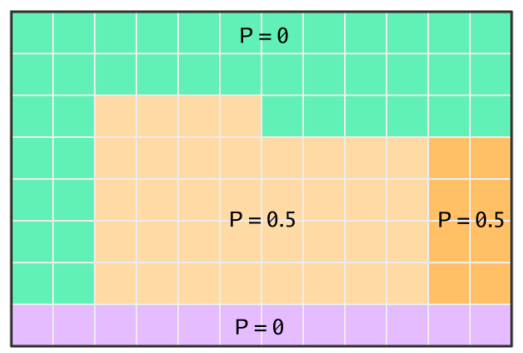

c) Dasymetric Dot Placement

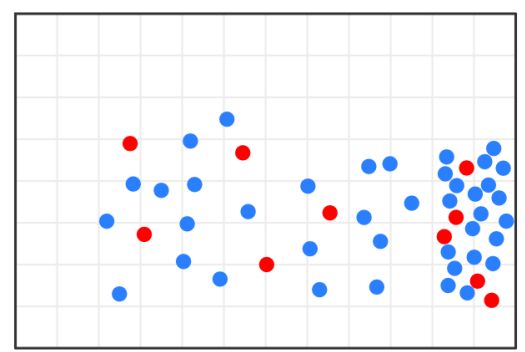

Figure 2: Schematic of how to place dots on dot maps (left: random locations, right: via dasymetric mapping)

Once the dots are generated for each time period in which we have known data, we can then estimate maps for intermediary time periods. This process is described both via the following text as well as visually in the schematic displayed in Figure 3.

Figure 3 A) displays, for a hypothetical example, the number of dots we have estimated for an area across for four data collection periods. One of the analytical limitations of demographic data is that they are typically collected at regular, but spaced out, intervals (e.g. every 5 years). However, if we want to view or display data over time, it is desirable to interpolate data for intermediate time periods. This can allow for more probable animations, transitions of change, or specific temporal queries (Bach, Dragicevic, Archambault, Hurter, \& Carpendale, 2014).

The standard approach for temporal interpolation of demographic data is estimating a continuous function, $P_{t}=f(t)$, which estimates the population, $P_{t}$, at a time period, $t$, fitted from a sample of known input 

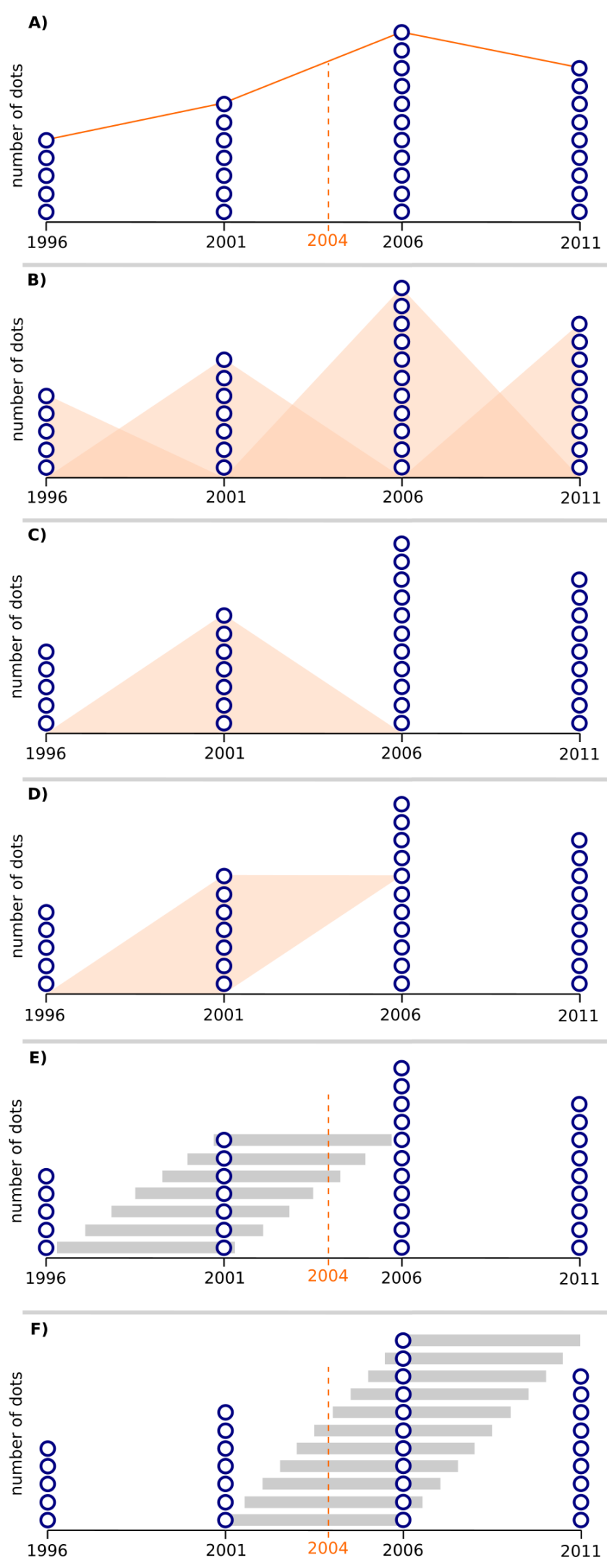

Figure 3: Schematic indicating temporal interpolation of dots on dot maps 
values at $t^{*}$. For example, this can assume an exact linear interpolation process, visually exemplified as the orange line in Figure $3 \mathrm{~A})$. With this function, $P_{t}=f(t)$, we can estimate dot maps for each data collection year. Using a linear, cumulative values approach, this would be as follows: $P_{t}=P_{i}+\frac{\left(t-t_{i}\right)\left(P_{j}-P_{i}\right)}{t_{j}-t_{i}}$ where $t=i$ and $t=j$ are the known anchor points, and $t$ is a time period between $i$ and $j$. However, it would be quite resource heavy and time-consuming to generate a completely new set of dots for every time period, $t$, we are interested in displaying, depending on how detailed we want our analysis to be. Moreover, doing this could also be visually discordant, as dot locations could flicker and shift quite quickly (Monmonier, 1996).

Our proposed solution to reduce this excess computation and flickered animation is to instead separate the interpolated function by the amount contributed by each year. In other words, take the interpolated function, $P_{t}=f(t)$ and decompose it into parts, $p_{t}=g(t)$ such that $\sum p_{t}=P_{t}=f(t)=\sum g(t)$, where each $g(t)$ is at its maximum at known data collection periods, $t^{*}$. This is visualized in Figure 3 B). The next step is to isolate each of these components, $g(t)$, like in Figure $3 \mathrm{C}$ ), and then transform it into a parallelogram as in Figure 3 D). This parallelogram has the same area as in Figure 3 C), with a base equal to the intervals between data collection period, and a height of $P_{t^{*}}$. This parallelogram can then be converted into a stepped shape, based on the number of the pre-selected rate of persons per dot.

The result of this process is shown in Figure $3 \mathrm{E}$ ) as a set of grey bars. Each grey bar in this stepped process represents the time period in which each dot is displayed on the map for. All of the bars are the same length if the intervals between data collection periods are stable (in this example, every 5 years). Each dot is coded with a unique time for when it first appears on the map, and a time when it no longer appears on the map. For any specific time, $t$, the dots can be set to be displayed with a simple filtering query, whether or not the selected time, $t$, falls within their respective ranges. So for the example of the year 2004, 3 of the dots generated from 2001 would be displayed, and the other 4 hidden, as shown in Figure $3 \mathrm{E}$ ). This strategy can then be repeated for each data collection period. Furthering this example of 2004 in Figure 3 F), 6 of the 11 dots generated from 2006 would be displayed on the map, for a combined number of displayed dots for a 2004 map being $3+6=9$.

Computing the total number of dots, $N_{t}$, for any time period, $t$, located between two anchor points $t=i$ and $t=j$ can be formalized as follows:

$$
N_{t}=\left[N_{i} \frac{t-t_{i}}{t_{j}-t_{i}}+N_{j} \frac{t_{j}-t}{t_{j}-t_{i}}\right]
$$

Importantly, as shown in Figure $3 \mathrm{E}$ ) and F), this processes allows for selecting a map for any time period without having to re-estimate the locations of points. This would save computation time, particularly for maps which include a lot of dots. Moreover, if animating change over time, this procedure also allows dots to gradually to appear and disappear from a map, without distracting flicker. For example, when transitioning from 2004 to 2006 as shown in Figure 3, 6 of the 9 dots on the map would remain in place (the bottom 6 pertaining to 2006), while 3 would disappear (the top 3 pertaining to 2001), and 5 new dots (the top 5 pertaining to 2006) would be added to the map.

This procedure does come with the assumption that for every time interval in which new data are made available, the population is refreshed and pertains to a new group of people. However, in reality, people do not move every $X$ years. Some people stay living in the same place for decades, while others move multiple times between new-data anchor time points. These characteristics are difficult to discern particularly when using demographic data that is only released as aggregated to spatial boundaries (i.e. when individual panel data is unavailable). This also becomes complicated when working with some categorical data (e.g. education levels, income categories, etc.) as some people may not move, but they may change which category they belong to. Improving our method to account for varying forms of mobility (spatial and categorical) would certainly be a worthwhile direction for future work, however, this would likely require 
access to hard-to-get disaggregate data to do this accurately.

\section{Applications}

We exemplify this procedure via two use case scenarios. The first examines population growth in the United States from 1900 to 2000, and the second explores changing distributions of poverty in Toronto, Canada from 1991 to 2016. These two scenarios were selected to show how this method can be applied across two different geographic scales, national and local, as well as for two different types of use, an animated map designed for a brief viewing time, and an interactive tool designed for nuanced exploratory analysis of a specific subject matter and locale.

\subsection{Quick Animation: USA Population Change}

In the first example, we create a short animation showing population growth across the USA during the 20th century. Data for this map are from historical tabulations of county level population data from the decennial United States census (Schroeder, 2016). A target-density dasymetric method was used to place dots, based on the population distributions in 2000 census tracts. Population data at a local scale was unavailable for earlier census years. Target-density dasymetric methods have been used previously in areal interpolation projects (Schroeder, 2007). Not incorporating this process would mean that dot generation would have to rely solely on county boundaries. In some counties, this would suffice, but in others, dots could be placed in areas that are clearly rural or remote. Los Angeles county, for example, had a population of 9.5 million in 2000, but more than half of its land area are mountains and desert (e.g. see the large size of the counties in the southwest in Figure 1)

This animated dot map is specifically designed for quick consumption on the internet. In terms of layout, the resolution of the map is 1920x1080 pixels, a standard screen size and aspect ratio. Each dot on the map represents 100 people, each visualized at a very minute size, but when combined, they highlight centres

of population. Dots are displayed over time using the selective interpolation procedure described in the previous section. The text on the layout of the map was kept to be minimal, to keep the focus on the map itself. The only other chart component is a bar at the bottom which represents the total population across the USA. The map can be viewed online (https://jamaps.github.io/D4M/usa/usa-pop-1990-to-2000 .gif). Figure 4 displays some of the frames of this animation as small multiples.

The map indicates spatial patterns of population growth, both at a national scale, as well as at more regional scales. In terms of national trends, the map shows more growth in the southern states and the west coast compared to the north-east of the USA during the 20th century. The map also conveys patterns of urban sprawl, which is particularly evident in urban regions like Los Angeles, the Bay Area, Atlanta, and Miami. Interesting as well is that the timing of urban sprawl varies. For example, Los Angeles appears to have substantial growth in the early 1900s, while urban sprawl in Florida occurs later in the 20th century.

\subsection{Interactive Exploration: Poverty Patterns in Toronto}

The second, and more extensive, example is an interactive application allowing for exploratory visual analysis of changing distributions of poverty in Toronto, a topic which has been a primary concern to a number of researchers (Ades et al., 2012; Hulchanski, 2010; Walks, 2013), particularly in relation to 


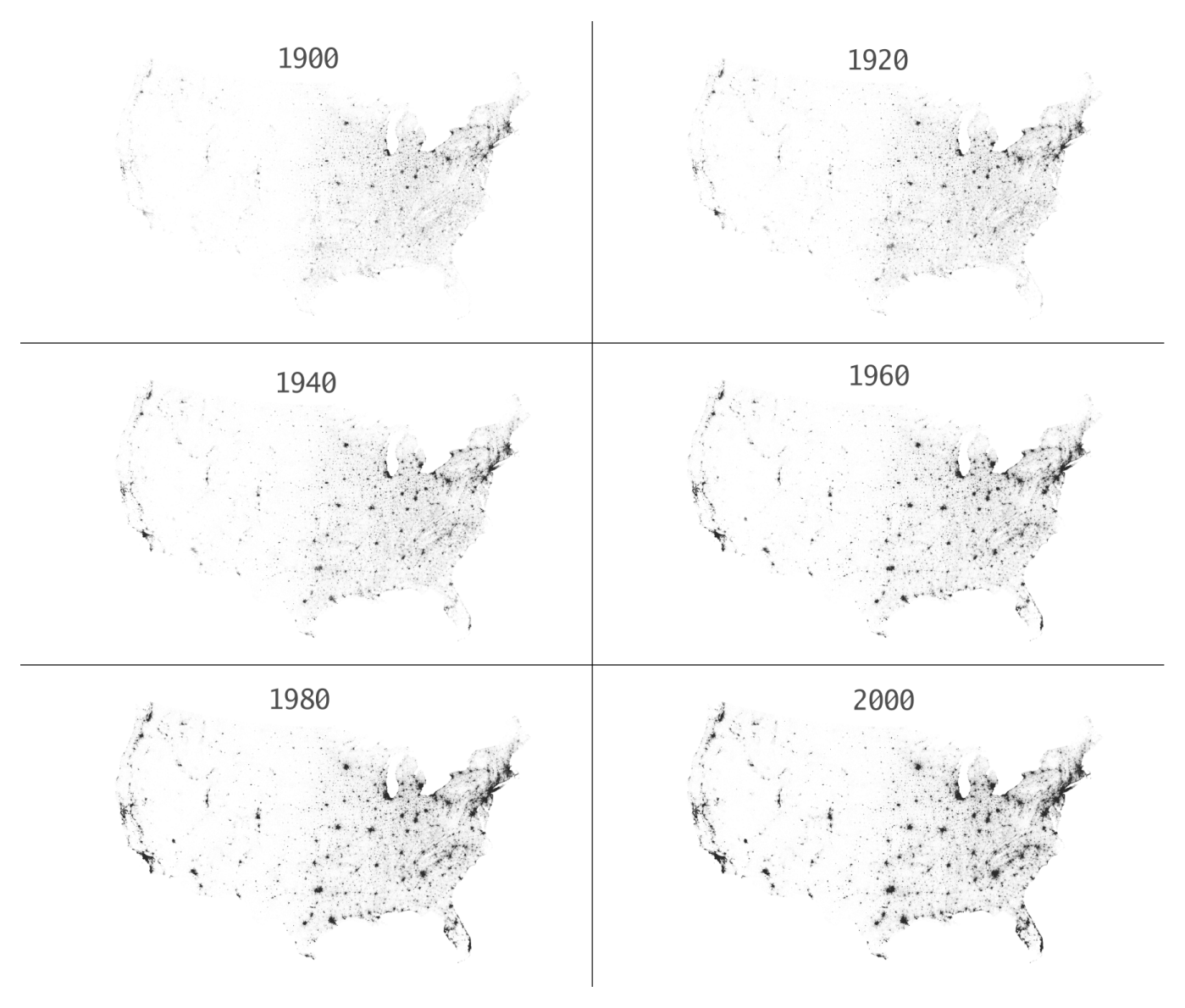

Figure 4: Selection of an animated dot map showing population change in the USA

problems of housing affordability (Skaburskis, 2012), available public transit (Allen \& Farber, 2019), and political polarization (Taylor, 2011).

Data for this map were drawn from six waves of the Canadian census, every five years from 1991 to 2016 (Statistics Canada, 2016). Data pertaining to different socio-economic categories were acquired as pre-aggregated to neighbourhood level spatial units. Given the local scale of analysis, two datasets were used to try to dasymetrically place dots on the map, in order to visualize local-level variations; land cover data from OpenStreetMap and block-level population counts. Using this auxiliary data, each input neighbourhood zone, $I$, were divided into sub-zones, $i$. Each, $i$, were then given a weighting probability of whether a person or household is located within it, $w_{i}$, such that $\sum_{i \in I} w_{i}=1$. The sub-zone of where dots are placed is then based on the discrete probability distribution of $I$. Dots were generated for each of the data collection years (1991, 1996, 2001, 2006, 2011, and 2016), and then interpolated for every year within this range using the selective interpolation method detailed in the previous section and Figure 3. A neighbourhood-level example of the result is displayed in Figure 5.

The data were then incorporated into an interactive map designed for exploratory visual analysis. The interactive map is available online (https://jamaps.github.io/D4M/toronto/index.html). Specifically, this interactive map was designed for researchers to be able to conduct the following five tasks:

1. Allow users to pan the map as well as zoom in and out in order view different regions and at different scales.

2. Provide a few categories of socio-economic status which can be selected for view on the map, as 
well as a "plain" map just showing the overall distribution of population.

3. Include the option to select data for different time periods, via a time slider bar.

4. Allow users to switch between dot density maps and choropleth maps showing the same variables.

5. Allow users to select regions on the map and display simple statistics for their selection.

Moreover, these tasks, allow for exploring the results of our procedure. Task (3) directly allows for transitioning dot maps over time, while tasks (1) and (2) allow for showing how the characteristics of such transitions vary for different scales and demographic categories, respectively. Task (4) provides users the ability to compare how transitioning dot maps over time fare relative to using a choropleth map for the same data and time periods. Task (5) simply provides users the ability to examine values for specific regions on the map, and how these values change over time, which can be compared with what is displayed on the map.

The layout of the application is divided into two parts. On the right, and taking up the majority of the space, is the interactive map. This is composed of several layers. The first is a basemap, which provides geographic reference, including streets, non built-up area, and water features. The colours and features on this basemap were selected to be faint and muted in order to not distract from the other layers, the overlaid demographic data.

The colours of the dot layer were selected to be blue and red because they stand out from each other and from the relatively white background. The red is chosen to indicate the lower socio-economic category, given the cultural association between red and a negative outcome. The map provides the ability for users to zoom, pan, and select different zones on the map. When zooming in and out, the number of dots visible changes, in order to limit dot overlap (Walker, 2018; Zhang et al., 2016). At a neighbourhood level, the number of people per dot is 50 , while zoomed out to a regional level, the number of people per dot is 200.

The data for the choropleth layer comes from a harmonized census tract database of Canadian cities (Allen \& Taylor, 2018). The breaks for the choropleth were based on quantiles, and then rounded to provide easier reading of the data. There are displayed as a sequential colour scheme, again, using a darker red to indicate impoverished areas.

On the left side of the application is a side-panel providing options to query for tasks (2)-(5). The time period can be queried via a time slider bar. At the bottom of this panel is a simple line chart showing the change in the selected variables over time, based on the selection made on the map. Selections can be made at the neighbourhood level as well as for municipal boundaries, which are often used in regional policy studies.

Overall, the temporal components of the map convey patterns of urban sprawl as well as intensification of development in centrally located neighbourhoods such as along the city's waterfront. As well, the map also indicates a slow growth of low-income households in more inner-suburban areas, but a consistency in clusters of zero-car households being located in the city centre. Much of these trends align with previous research (Ades et al., 2012; Sun, 2019)

\section{Conclusions}

This paper detailed a procedure for extending dot maps so they can visualize changes in spatial patterns over time. Specifically, a selective linear interpolation procedure was developed to encode the time range in which dots are visible on a map. This methodology was exemplified first by animating population growth across the United States during the 20th century, and second, through an interactive application 


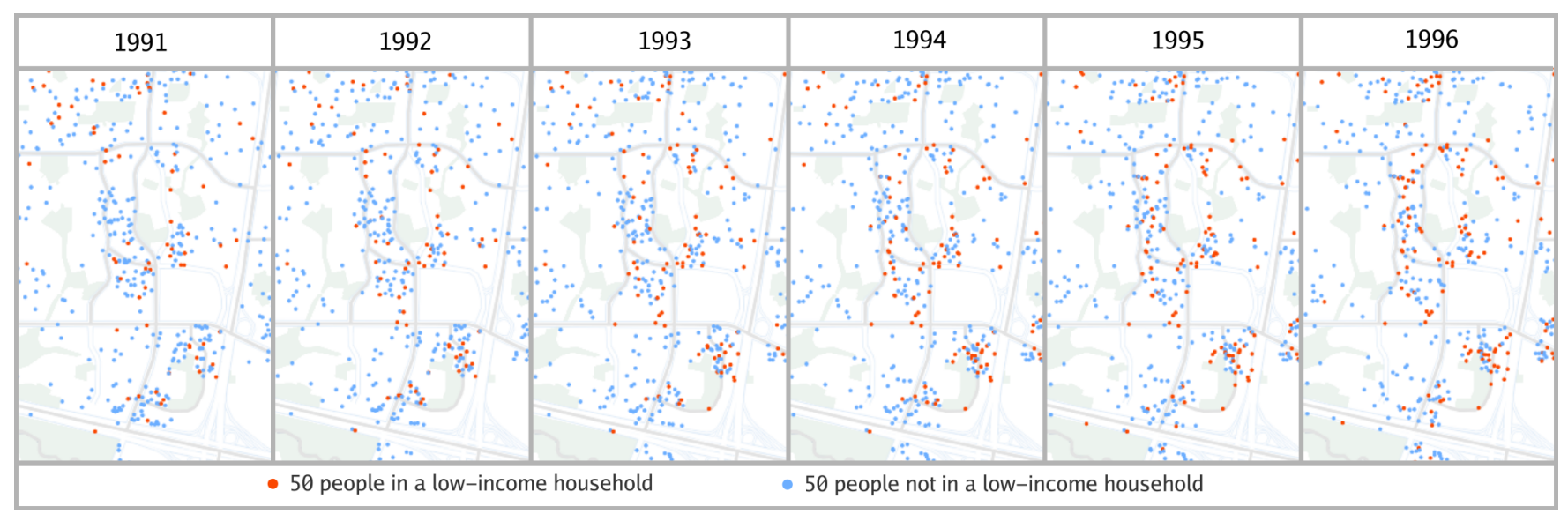

Figure 5: Selection of an interpolated dot map showing increasing poverty concentrations in a neighbourhood in Toronto

showing changing poverty distributions in Toronto, Canada. The maps indicate multi-scale patterns of population change, and in the case of the second example, shifting distributions of socio-economic status.

One limitation of this work is that these dot maps do not show to the user the uncertainty of dot placement nor that of the temporal interpolation process. Future work could explore how such uncertainty could be visualized to users. One possible solution could be to incorporate a kernel density surface indicating approximate distributions of populations as a faint layer under the dot density map.

Future work could also extend temporal interpolation of dot maps to similar types of thematic maps, such as graduated dot maps (Arnold, Jenny, \& White, 2017; Gomes, 2017) or dot map cartograms (Soetens, Hahne, \& Wallinga, 2017). However, in some of these maps, the locations of dots are often geographically shifted or distorted, and it would be more complicated to animate changes over time, compared to when the geographical reference remains consistent.

Lastly, another avenue for future work would be to conduct detailed user evaluations of dynamic dot maps. One direction would be to examine how the design of transitions between time periods could be optimized. Another direction would be to evaluate how temporal dot density maps, like those presented in this paper, fare against other types of thematic maps such as choropleths, in terms of knowledge translation. This could either be conducted via quantitative recall experiments or through qualitative preference questionnaires and semi-structured interviews.

\section{References}

Ades, J., Apparicio, P., \& Seguin, A.-M. (2012). Are new patterns of low-income distribution emerging in Canadian metropolitan areas? The Canadian Geographer/Le Geographe canadien, 56(3), 339-361.

Allen, J., \& Farber, S. (2019). Sizing up transport poverty: A national scale accounting of low-income households suffering from inaccessibility in Canada, and what to do about it. Transport Policy, 74, 214-223.

Allen, J., \& Leahey, A. (2016). Improving access to digital historical census boundaries in canada. ACMLA Bulletin(153), 44-50. 
Allen, J., \& Taylor, Z. (2018). A new tool for neighbourhood change research: The Canadian Longitudinal Census Tract Database, 1971-2016. The Canadian Geographer/Le Geographe canadien, 62(4), 575-588.

Arnold, N. D., Jenny, B., \& White, D. (2017, December). Automation and evaluation of graduated dot maps. International Journal of Geographical Information Science, 31(12), 25242542. Retrieved from https://doi.org/10.1080/13658816.2017.1359747 doi: doi: 10.1080/13658816.2017.1359747

Bach, B., Dragicevic, P., Archambault, D., Hurter, C., \& Carpendale, S. (2014, June). A Review of Temporal Data Visualizations Based on Space-Time Cube Operations.. Retrieved from https://hal.inria.fr/hal-01006140

Barreto, M. L. (1993, August). The Dot Map as an Epidemiological Tool: A Case Study of Schistosoma mansoni Infection in an Urban Setting. International Journal of Epidemiology, 22(4), 731-741. Retrieved from https://academic.oup.com/ije/article/22/4/731/664119 doi: doi: $10.1093 /$ ije/22.4.731

Birch, T. (1938). Dotting The Dot Map. In Notes on Statistical Mapping. American Geographical Society.

Cable, D. (2013). The Racial Dot Map. Weldon Cooper Center for Public Service, University of Virginia. Retrieved from https://demographics.virginia.edu/DotMap

Chen, C. (2010). Information visualization. WIREs Computational Statistics, 2(4), 387-403. Retrieved from https://onlinelibrary.wiley.com/doi/abs/10.1002/wics.89 doi: doi: $10.1002 /$ wics. 89

Chevalier, F., Dragicevic, P., \& Franconeri, S. (2014, December). The Not-so-Staggering Effect of Staggered Animated Transitions on Visual Tracking. IEEE Transactions on Visualization and Computer Graphics, 20(12), 2241-2250. doi: doi: 10.1109/TVCG.2014.2346424

Chi, G., \& Voss, P. R. (2011). Small-area population forecasting: borrowing strength across space and time. Population, Space and Place, 17(5), 505-520. Retrieved from https:// onlinelibrary.wiley.com/doi/abs/10.1002/psp.617 doi: doi: 10.1002/psp.617

Delmelle, E. C. (2017). Differentiating pathways of neighborhood change in 50 US metropolitan areas. Environment and planning A, 49(10), 2402-2424.

Dent, B., Torguson, J., \& Hodler, T. (1990). Cartography: Thematic Map Design. Wm. C. Brown Publishers.

Dmowska, A., \& Stepinski, T. F. (2019, May). Racial Dot Maps Based on Dasymetrically Modeled Gridded Population Data. Social Sciences, 8(5), 157. Retrieved from https:// www.mdpi.com/2076-0760/8/5/157 doi: doi: 10.3390/socsci8050157

Dorling, D. (1993, December). Map design for census mapping. The Cartographic Journal, 30(2), 167-183. Retrieved from https://www.tandfonline.com/doi/abs/10.1179/ 000870493787860175 doi: doi: 10.1179/000870493787860175

Franklin, R. S. (2019, July). The demographic burden of population loss in US cities, 2000-2010. Journal of Geographical Systems. Retrieved from https://doi.org/10.1007/s10109-019 -00303-4 doi: doi: 10.1007/s10109-019-00303-4

Frere de Montizon, A. J. (1830). Carte philosophique figurant la population de la France.

Fukuda, K. (2010). Interpolation and forecasting of population census data. Journal of Population Research, 27(1), 1-13.

Gehlke, C. E., \& Biehl, K. (1934, March). Certain Effects of Grouping upon the Size of the Correlation Coefficient in Census Tract Material. Journal of the American Statistical Association, 29(185A), 169-170. Retrieved from https://doi.org/10.1080/01621459.1934.10506247 doi: doi: 10.1080/01621459.1934.10506247

Gomes, E. (2017). Creating a Dot Density Map: Resident Population in Mainland Portugal. The Cartographic Journal, 54(2), 157-162. Retrieved from http://journals.scholarsportal 
.info/detailsundefined doi: doi: 10.1080/00087041.2016.1148106

Hey, A. (2012, January). Automated Dot Mapping: How to Dot the Dot Map. Cartography and Geographic Information Science, 39(1), 17-29. Retrieved from https://doi.org/10.1559/ 1523040639117 doi: doi: 10.1559/1523040639117

Hey, A., \& Bill, R. (2014). Placing dots in dot maps. International Journal of Geographical Information Science, 28(12), 2417-2434. Retrieved from http://journals . scholarsportal.info/ detailsundefined doi: doi: 10.1080/13658816.2014.928822

Holt, J. B., Lo, C. P., \& Hodler, T. W. (2004, January). Dasymetric Estimation of Population Density and Areal Interpolation of Census Data. Cartography and Geographic Information Science, 31(2), 103-121. Retrieved from https://doi.org/10.1559/1523040041649407 doi: doi: $10.1559 / 1523040041649407$

Hulchanski, J. D. (2010). The three cities within Toronto. Cities Centre: University of Toronto.

Kimerling, A. J. (2009, January). Dotting the Dot Map, Revisited. Cartography and Geographic Information Science, 36(2), 165-182. Retrieved from https://doi.org/10.1559/ 152304009788188754 doi: doi: 10.1559/152304009788188754

Konvitz, J. (1987). Cartography in France, 1660-1848: Science, Engineering, and Statecraft. University of Chicago Press.

Lewandowsky, S., Herrmann, D. J., Behrens, J. T., Li, S.-C., Pickle, L., \& Jobe, J. B. (1993). Perception of clusters in statistical maps. Applied Cognitive Psychology, 7(6), 533-551. Retrieved from https://onlinelibrary.wiley.com/doi/abs/10.1002/acp.2350070606 doi: doi: 10.1002/acp.2350070606

Logan, J. R., Xu, Z., \& Stults, B. J. (2014, July). Interpolating U.S. Decennial Census Tract Data from as Early as 1970 to 2010: A Longitudinal Tract Database. The Professional Geographer, 66(3), 412-420. Retrieved from https://doi.org/10.1080/00330124.2014.905156 doi: doi: $10.1080 / 00330124.2014 .905156$

MacEachren, A. M. (2004). How Maps Work: Representation, Visualization, and Design. Guilford Press.

MacEachren, A. M., \& DiBiase, D. (1991, January). Animated Maps of Aggregate Data: Conceptual and Practical Problems. Cartography and Geographic Information Systems, 18(4), 221-229. Retrieved from https://doi.org/10.1559/152304091783786790 doi: doi: $10.1559 / 152304091783786790$

Mackay, R., J. (1949). Dotting the dot map: An analysis of dot size, number, and visual tone density. Surveying and Mapping, 9(1), 3-10.

Missiakoulis, S. (2010). Cecrops, King of Athens: the First (?) Recorded Population Census in History. International Statistical Review, 78(3), 413-418. Retrieved from https:// onlinelibrary.wiley.com/doi/abs/10.1111/j.1751-5823.2010.00124.x doi: doi: 10.1111/j.1751-5823.2010.00124.x

Monmonier, M. (1996). Temporal Generalization for Dynamic Maps. Cartography and Geographic Information Systems, 23(2), 96-98. Retrieved from http://journals.scholarsportal . info/detailsundefined doi: doi: 10.1559/152304096782562118

Openshaw, S. (1979). A million or so correlation coefficients : three experiments on the modifiable areal unit problem. Statistical applications in the spatial science, 127-144.

Park, D., Drucker, S. M., Fernandez, R., \& Elmqvist, N. (2018, December). Atom: A Grammar for Unit Visualizations. IEEE Transactions on Visualization and Computer Graphics, 24(12), 3032-3043. doi: doi: 10.1109/TVCG.2017.2785807

Petermann, A. (1852). England and Wales: distribution of the population. Census of Great Britain,1851,Population Tables I. Her Majesty's Stationery Office, London.

Petrov, A. (2012, August). One Hundred Years of Dasymetric Mapping: Back to the Origin. The Cartographic Journal, 49(3), 256-264. Retrieved from https://doi.org/10.1179/ 
1743277412 Y.0000000001 doi: doi: 10.1179/1743277412Y.0000000001

Schroeder, J. P. (2007). Target-Density Weighting Interpolation and Uncertainty Evaluation for Temporal Analysis of Census Data. Geographical Analysis, 39(3), 311-335. Retrieved from https://onlinelibrary.wiley.com/doi/abs/10.1111/j.1538-4632. 2007 .00706.x doi: doi: 10.1111/j.1538-4632.2007.00706.x

Schroeder, J. P. (2016). Historical Population Estimates for 2010 U.S. States, Counties and Metro/Micro Areas, 1790-2010. Data Repository for the University of Minnesota. Retrieved from http://conservancy.umn.edu/handle/11299/181605 (type: dataset) doi: doi: http://doi.org/10.13020/D6XW2H

Skaburskis, A. (2012). Gentrification and Toronto's changing household characteristics and income distribution. Journal of Planning Education and Research, 32(2), 191-203.

Soetens, L., Hahne, S., \& Wallinga, J. (2017, June). Dot map cartograms for detection of infectious disease outbreaks: an application to $Q$ fever, the Netherlands and pertussis, Germany. Eurosurveillance, 22(26). Retrieved from https://www.ncbi.nlm.nih.gov/pmc/ articles/PMC5779165/ doi: doi: 10.2807/1560-7917.ES.2017.22.26.30562

Statistics Canada. (2016). Dictionary, Census of Population, 2016. Retrieved from https:// www12.statcan.gc.ca/census-recensement/2016/ref/dict/index-eng.cfm

St-Hilaire, M., Moldofsky, B., Richard, L., \& Beaudry, M. (2007, April). Geocoding and Mapping Historical Census Data: The Geographical Component of the Canadian Century Research Infrastructure. Historical Methods: A Journal of Quantitative and Interdisciplinary History, 40(2), 76-91. Retrieved from https://doi.org/10.3200/HMTS.40.2.76-91 doi: doi: 10.3200/HMTS.40.2.76-91

Sun, X. (2019). Empirical Analysis of Urban Sprawl in Canadian Census Metropolitan Areas using Satellite Imagery, 1986-2016 (Doctoral dissertation, Western University). Retrieved from https://ir.lib.uwo.ca/etd/6394

Taylor, Z. (2011). Who Elected Rob Ford, and Why? An Ecological Analysis of the 2010 Toronto Election. Waterloo, ON.

Turner, E., \& Allen, J. P. (2010). Issues in Depicting Population Change with Dot Maps. Cartography and Geographic Information Science, 37(3), 189-197. Retrieved from http://journals . scholarsportal.info/detailsundefined doi: doi: 10.1559/152304010792194921

Vasiliev, I. (2006, September). Mapping Time. Cartographica: The International Journal for Geographic Information and Geovisualization. Retrieved from https://www.utpjournals . press/doi/abs/10.3138/D357-234G-2M62-4373 doi: doi: 10.3138/D357-234G-2M62 $-4373$

Walker, K. E. (2018, October). Scaling the Interactive Dot Map. Cartographica: The International Journal for Geographic Information and Geovisualization. Retrieved from https:// www.utpjournals.press/doi/abs/10.3138/cart.53.3.2017-0021 doi: doi: 10.3138/ cart.53.3.2017-0021

Walks, A. (2013). Income inequality and polarization in Canada's cities: An examination and new form of measurement. Cities Centre, University of Toronto.

Zhang, D., Zhu, L., Xiao, Z., \& Zhang, L. (2016, May). Visual abstraction improvement of interactive dot map. In 2016 17th IEEE/ACIS International Conference on Software Engineering, Artificial Intelligence, Networking and Parallel/Distributed Computing (SNPD) (pp. 469-474). doi: doi: 10.1109/SNPD.2016.7515942 